\title{
TELA, STOFFA, ABITO, O DEL METODO DELLA CREAZIONE NELLA POESIA CONTEMPORANEA FRANCESE (PONGE, BONNEFOY, FOURCADE)
}

\author{
Silvia Riva \\ UnIVERSITÀ DEgLI Studi di Milano
}

\begin{abstract}
Ayant un peu le goût des mots perdus, j'ai souhaité observer ici quelques figurations du poète, durant cette période dite moderne qui le voit précisément engager lui-même son propre procès. Et puisqu'il se portraiture volontiers en funambule, en sonneur de cloches, en pendu ou en araignée, on verra que ce sont pour beaucoup des affaires de cordes et de fils (d'encre) qui l'occupent. Perplexe, occupé à tisser des liens, penché sur son ouvrage plutôt que tourné vers l'Azur, le poète tardif est critique avant tout. Étudier ses figures, c'est dès lors s'inquiéter du maintien de la poésie; c'est interroger son pourquoi, son sens et sa valeur.
\end{abstract}

Jean-Michel Maulpoix, Le poète perplexe

Lo spunto offerto dal tema del vêtement, affrontato in queste pagine, invita ad interrogarsi su come la poesia contemporanea francese abbia attinto all'usata analogia fra testo e tessuto, suggerita dall'etimologia di textus, per riflettere in modo nuovo sui mezzi e metodi del suo stesso farsi.

I motivi della tela, certo, ma anche della stoffa e infine dell'abito consentono, infatti, di abbozzare un breve percorso sull'uso della metafora della costruzione di trame nella poesia dell'extrême contemporain e dei suoi immediati predecessori, che interroghi, come è detto nell'esergo di JeanMichel Maulpoix tratto dal saggio Le poète perplexe, non tanto ambiziosamente il suo valore, quanto piuttosto il senso del suo perché e del suo farsi.

Distanziandosi dalla tradizione degli Antichi, che già avevano ampiamente fatto ricorso all'immagine della tela in poesia associandola a una

I Maulpoix (2002: 386). 
riflessione sull'inserzione della voce del poeta nella filigrana dello scritto, gli autori del contemporaneo sono sempre meno 'perplessi', per riprendere l'idea suggerita nel titolo da cui è tratto il testo d'esordio, nell'assorbire e criticare la metafora del tessuto per illustrare il proprio scopo.

L'ipotesi alla base di queste brevi riflessioni è che la figura della tela, della trama e dell'abito non sia più (soltanto) usata per dar conto del lavoro del poeta, quanto piuttosto per riflettere sulla funzione da protagonista, se non da regista, rispettivamente, del lettore, del linguaggio e delle immagini nella prosodia di oggi.

Attraverso l'analisi dei tre elementi attinti alle opere e alle riflessioni di altrettanti esponenti della produzione poetica contemporanea (Francis Ponge, Yves Bonnefoy e Dominique Fourcade), ci chiederemo se la coppia analogica testo / tessuto si sia nel tempo consumata, oppure se abbia trovato nuove fogge per dar forma e dire il mondo.

\section{TELE Di RAgNo}

Il riconoscimento della fragilità, se non della nudità della relazione fra idee / cose e parole, è consustanziale alla creazione poetica; si può forse affermare che ne sia proprio il fondamento.

Francis Ponge è, fra gli autori del secondo Novecento, colui che, forse più di altri, assume appieno la consapevolezza di tale fragilità e, con lei, il conseguente rischio che corre la poesia di impoverire la sua portata se, in modo narcisistico, il poeta mira a ottenere effetti sui lettori (che destino ammirazione, ma anche sconcerto) al posto di attenersi alla restituzione della piatta materialità delle cose, nel loro presente concreto.

Fra le tante realtà che possono costituire l'oggetto della poesia e, contemporaneamente, che offrono spunto per riflettere sul suo fine (e oggetto), trovano posto anche il ragno e la tela. Non soltanto perché, come si è detto, textus rinvia tanto alla composizione di uno scritto quanto al risultato della (sua) tessitura, ma soprattutto se si ricorda, come lo stesso Ponge invita a fare e sulla scorta di quanto suggerisce il dizionario Littré ridando vita allo spessore semantico delle parole, che nella lingua di oggi è riunito ciò che nel Medio francese ancora era diviso, ossia l'aragne (l'artefice della tela) e l'araignée (il suo artefatto) ${ }^{2}$.

Prolungando (in francese si potrebbe dire anche 'filando') così, com'è consueto nella scrittura di Ponge, un'analogia che funziona attenendosi esclusivamente al piano referenziale dell'enunciazione che, tuttavia, come si è appena detto, è duplice, in «L'Araignée» sono illustrate le tappe successive della formazione di una tela / testo, cercando di mettere in luce, di volta in volta, sia le proprietà immanenti all'oggetto, sia le operazioni che l'aracnide / poeta compie tessendo con la sua bava.

\footnotetext{
2 Vedi su questo aspetto il capitolo «Ponge et Rameau», in Meadows (I997: 59 e sg.).
} 
La sintassi in cui si dipana la poesia mima dunque il procedimento di costruzione dell'opera.

Il poema inizia perciò con un exorde (nella cui etimologia oscilla, accanto all'etimo dell'inizio - ordium -, anche quello dell'ordito), in cui sono enunciati i movimenti (intesi anche in senso musicale) del ragno e del poeta, una lenta danza in tre tempi (una sarabanda, che sarà seguita da una fuga) grazie alla quale entrambi gli artefici riescono a fabbricare una rete in cui trattenere le loro prede, correndo su fili che essi stessi creano, come si trattasse di spole, in un senso e poi nel senso inverso, mettendo al centro il loro proposito, per poi abbandonarlo, passando ad altro, ma ripercorrendo a ritroso, uno per uno, tutti i fili (del discorso) già tesi:

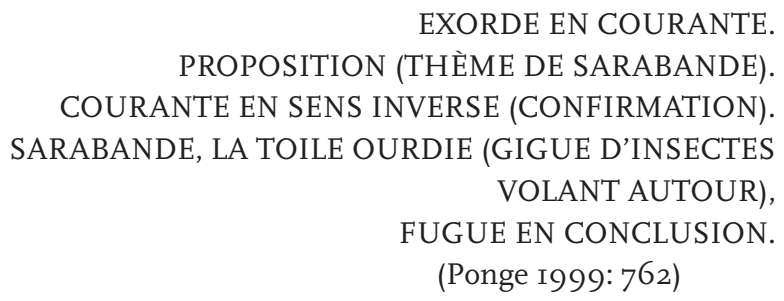

A nostra volta «bestioles» (Ibidem) (è in tal modo che l'io lirico definisce i malcapitati lettori / prede), assistiamo successivamente all'esposizione del metodo della tessitura di questi «propos en l'air» (763) nei quali rimaniamo impigliati.

\begin{abstract}
Mais d'abord, comment agit-elle?
Est-ce d'un bond hardi? Ou se laissant tomber sans lâcher le fil de son discours, pour revenir plusieurs fois par divers chemins ensuite à son point de départ, sans avoir tracé, tendu une ligne que son corps n'y soit passé - n'y ait tout entier participé - à la fois filature et tissage?

D'où la définition par elle-même de sa toile aussitôt conçue:

DE RIEN D'AUTRE QUE DE SALIVE PROPOS EN L'AIR MAIS AUTHENTIQUEMENT TISSUS - OU J'HABITE AVEC PATIENCE - SANS PRÉTEXTE QUE MON APPÉTIT DE LECTEURS.
\end{abstract}

(Ibidem)

Come ci ricorda l'eminente latinista Jesper Svenbro (Svenbro 2008: 24 sg.), già un oscuro poeta del VI secolo, Luxurius, aveva istituito nei suoi Carmina (288. Happ. Scheid-Svenbro I994: I58f) un parallelismo fra il testo e un tessuto all'interno del quale il lettore introdurrebbe (il verbo latino impiegato è retexere) la sua trama vocale: grazie a quest'ultima, ogni volta, l'opera si ricostruisce e si disfà quando il libro viene richiuso o la 
poesia smette di essere cantata. Tuttavia, Ponge non si limita a enunciare poeticamente l'interdipendenza fra autore e interpreti (di cui fa un lungo e dissacrante elenco in forma di bestiario); e nemmeno s'accontenta d'illustrare all'opera il principio della semiosi illimitata, che dà luogo a «scaramouches au ciel» (Ponge I999: 763) fra il poeta / ragno e le sue prede, che egli divorerà in silenzio introducendole nella sua grossa pancia:

\begin{abstract}
À son propos ainsi - à son image -, me faut-il lancer des phrases à la fois assez hardies et sortant uniquement de moi, mais assez solides - et faire ma démarche assez légère, pour que mon corps sans les rompre sur elles prenne appui pour en imaginer en lancer d'autres en sens divers - et même en sens contraire par qui soit si parfaitement tramé mon ouvrage, que ma panse dès lors puisse s'y reposer, s'y tapir, et que je puisse y convoquer mes proies - vous, lecteurs, vous, attention de mes lecteurs - afin de vous dévorer ensuite en silence (ce qu'on appelle la gloire)... (Ibidem)
\end{abstract}

Giocando, questa volta, sull'omofonia fra la terza persona del verbo «pensare» in francese e la citata panse (che, è, come ricorda il Littré, non solo ventre, ma anche un modo per dire «scrivere» ${ }^{3}$, e anche la prima parte dello stomaco di un ruminante; per non parlare poi del proverbio 'Après la panse vient la danse', sarabanda e pasto luculliano allo stesso tempo), Ponge espone, attraverso una mise en abyme ardita che risulta dall'utilizzo di numerose altre duplicazioni di forma-senso (ad esempio, nella citazione successiva, secretare e segreto, che in francese conservano la «c» in entrambi i casi; il gioco di parole fra la terza persona del verbo uscire (sortir) e la parola «sort» (764), destino; oppure il neologismo «échriveau» (Ibidem), crasi che gioca su molteplici livelli - tra cui la ripresa del ruminante in veau -, ma anche sulla fusione tra écrivain e écheveau, matassa in francese) il principio alla base della (sua) poesia, ossia il prevalere del significante (della materialità della parola, della sua parte puramente fisica) sul significato.

In altri termini, egli espone concretamente il trionfo della pratica poetica sulla metafisica cartesiana del 'penso quindi sono':

FREDONS, BILLEVESÉES, SCHÈMES EN ZIZANIE! SACHEZ, QUOI QU'IL EN SOIT DE MA PANSE SECRÈTE ET BIEN QUE JE NE SOIS QU'UN ECHRIVEAU CONFUS

3 Alla parola panse corrisponde, infatti, la definizione della lettera «a» nel Dictionnaire de la langue française «Littré» (che è, peraltro, la prima definizione in assoluto del voluminoso dizionario): «Une panse d'a, la première partie d'un petit a dans l'écriture cursive. N'avoir pas fait une panse d'a, c'est-à-dire n'avoir rien écrit, rien copié, rien composé» (Dictionnaire de la langue française «Littré»: s.v. a). 
QU'ON EN PEUT DÉMÊLER POUR L'HEURE CE QUI SUIT: À SAVOIR QU'IL EN SORT QUE JE SUIS VOTRE PARQUE; SORT, DIS-JE, ET IL S'ENSUIT QUE BIEN QUE JE NE SOIS QUE PANSE DONC JE SUIS (SACHET, COQUILLE EN SOIE QUE MA PANSE SECRÈTE) VOTRE MAUVAISE ÉTOILE AU PLAFOND QUI VOUS GUETTE POUR VOUS FAIRE EN SES RAIS CONNAÎTRE VOTRE NUIT. (Ibidem; corsivi miei)

La missione del poeta è dunque quella di far conoscere all'uomo, preso nei raggi e nella rete del discorso, nella danza del cosmo o nella matassa di seta, la sua notte, la sua fine (e il suo fine). Ecco perché il ragno / poeta è come le Parche del mito.

Tuttavia, non si tratta di una notte oscura e la fine è soltanto provvisoria, tanto per l'artefice, quanto per la preda:

Beaucoup plus tard, - ma toile abandonnée - de la rosée, des poussières l'empèseront, la feront briller - la rendront de toute autre façon attirante...

Jusqu'à ce qu'elle coiffe enfin, de manière horrible ou grotesque, quelque amateur curieux des buissons ou des coins de grenier, qui pestera contre elle, mais en restera coiffé.

Et ce sera la fin...

Mais fi!

De ce répugnant triomphe, payé par la destruction de mon œuvre, ne subsistera dans ma mémoire orgueil ni affliction, car (fonction de mon corps seul et de son appétit) quant à moi mon pouvoir demeure!

Et dès longtemps, - pour l'éprouver ailleurs - j'aurai fui... (765; corsivi miei)

Ciò che rimane costante di opera in opera è, infatti, il potere della materialità della lingua, che deve essere, ogni volta, reinventata.

È questo 'metodo sul discorso poetico' che Ponge illustra concretamente in «L'Araignée» e che egli riprende in uno scritto successivo, in cui, nuovamente, il poeta sottolinea la capacità della parola di ricreare e abbracciare il mondo quando ci si attiene alla sola dimensione fisica della materia e si comprende il valore salvifico anche nella distruzione e confusione nella realtà:

La fonction de la poésie [...] est de nourrir l'esprit de l'homme en l'abouchant au cosmos. Il suffit d'abaisser notre prétention à dominer la nature et d'élever notre prétention à en faire physiquement partie, pour que la réconciliation ait lieu. Quand l'homme sera fier d'être non seulement le lieu où s'élaborent les idées et 
les sentiments mais aussi bien le nœud où ils se détruisent et se confondent, il sera prêt alors d'être sauvé. L'espoir est donc dans une poésie par laquelle le monde envahisse à ce point l'esprit de l'homme qu'il en perde à peu près la parole, puis réinvente un jargon. (Ponge i96r: I98)

La reinvenzione del linguaggio ci conduce così a una successiva figura appartenente alla costellazione semantica del vêtement. Si tratterà ora di stoffa e, di nuovo, della ricerca di un metodo per dire la nudità della relazione fra idee / cose e parole. Una nudità, uno stato di natura e innocenza, che, per contrasto, l'uso della stoffa ancor più sottolinea.

\title{
2. Di STOFFA O D'ARGILLA?
}

L'opera di Yves Bonnefoy è immensa, per importanza, articolazione e ampiezza.

Nello spazio a disposizione ci si atterrà esclusivamente alla lettura di una prefazione a un libro di Patrice Hugues, uscito nel I996, intitolato Tissu et travail de civilisation. La prefazione, scritta dal poeta di Tours in quell'occasione, è stata ripresa in un volume uscito nel 2010, pubblicato nei «Carnets de l'Herne», col titolo Pensées d'étoffe ou d'argile, nel quale la presentazione è associata ad alcune pagine, profondamente rimaneggiate in quest'ultima versione, che erano state scritte nel I976 in occasione di un'esposizione del ceramista Norbert Pierlot (e riprodotte poi nel I989, nel volume Sur un sculpteur et des peintres).

In Pensées d'étoffe ou d'argile, dunque, Bonnefoy esce dall'identificazione testo / tessuto per accostare piuttosto la stoffa al linguaggio e alla sua ambivalente capacità dialettica.

Egli invita, innanzitutto, ad andare al di là delle considerazioni culturali relative al tessuto e ai segni che in esso sono inscritti:

\begin{abstract}
à ces derniers [...] si on s'y intéresse, c'est presque toujours pour eux-mêmes, c'est-à-dire en oubliant leur substrat pour se placer dans la perspective toute autre des formes qu'une culture élabore à tous les points à la fois de son invention artistique ou des significations dont elle a besoin pour appréhender l'univers. On regarde passer les barques, on méconnaît qu'il y a pour les porter, pour les guider peut-être, un grand fleuve. (Bonnefoy 20I0: I5-I6)
\end{abstract}

È il sostrato sotterraneo ciò che per lui più conta. E se si sondano le origini della nascita di questo manufatto e s'indagano le sue caratteristiche più segrete, ci si accorge che c'è qualcosa di sacro, quindi di ambivalente nella «déesse du textile» (I6). 
In primo luogo, Bonnefoy ci ricorda come, fin dall'Antichità, il filo del tessuto sia presente nei momenti più lieti della rivelazione del divino (ad esempio nell'arte bizantina, che sempre ritrae, nell'attimo dell'Annunciazione, l'angelo che coglie Maria intenta a filare la lana), quanto nei momenti di terrore. Tornano le Parche, per le quali «on identifie leur fil au temps qui entraîne dans l'inconnu» (I7), ma anche «l'araignée, que l'on n'aime pas, [qui] tisse sa toile» (Ibidem).

Sempre in modo ambivalente, il tessuto è associato anche a ciò che, diabolicamente, vela o nasconde, «comme si la première fonction de ce qui devint la vêture était de cacher la première faute» (I8) di Adamo ed Eva; ma esso ha a che fare anche con ciò che si mostra nella sua totale, lieta innocenza, come «l'enfant dans ses premières saisons» (Ibidem) o, divinamente, come il volto del Cristo impresso nel velo di Veronica.

Soprattutto, l'elemento più sorprendente che la stoffa cela in sé è «aussi ce qui soi-même se cache» (Ibidem): in particolare, la sua potenziale infinitezza (che l'accosta di nuovo al divino) e la sua capacità di essere tanto più invisibile (e obliata) quanto più ci è vicina (e presente):

Le tissu n'a pas, comme tel, de délimitation naturelle, il pourrait continuer sans fin au-delà de tout écheveau. (Ibidem)

\section{E ancora:}

[Le tissu] existe au revers de l'existence, dans une dimension en somme impensable et qu'à chaque instant, et si même on la pressent, on l'oublie. Ce qui fait que lui qui est né par rupture avec la nature, celle que Dieu a voulue, et peut passer ainsi pour un des moyens du diable: la capacité d'être invisible en même temps que tout proche. (I8-19)

Ecco perché più che al testo, il tessuto fa pensare al linguaggio («ce lieu originel de la rupture de l'être humain avec la simple nature»: 20) e alla sua ambiguità sostanziale. Un'ambivalenza che il poeta rileva ancor più viva laddove il linguaggio prende forma di utopia, ossia laddove è operante il sogno che la parola possa disfarsi, una volta per tutte, della sua essenziale disonestà («capacité de tromper»: 2I).

Così, ogni volta che si è indotti utopisticamente ad affidarsi al linguaggio, ci si affida anche alla verità dell'abito:

chaque fois [...] qu'on estime qu'on n'a plus à craindre l'emploi des mots, on regarde l'étoffe aussi avec une confiance nouvelle, à supposer qu'en aient disparu toutes les marques et tous les signes qui ne seraient pas ceux de la pensée officielle. (Ibidem) 
Gli esempi delle divise maoiste, tutte uguali e fabbricate con la medesima stoffa, o dei jeans tagliati, che intrattengono l'illusione che il corpo possa trionfare sulle alienazioni che colpevolizzano la nudità, insite nel pensiero occidentale, ne sono un chiaro emblema e, nella penna di Bonnefoy, diventano persino oggetto d'ironia: dei jeans lacerati dice, infatti, «avec ce genou qui se montre dans l'échancrure du bleu, plus besoin de se souvenir du voile de Véronique» (22).

Il che lo induce a concludere che il tema della stoffa sia essenziale: «la question du textile est fondamentale, à peu près autant que celle de la parole, à peu près autant que celle de l'être» (Ibidem).

Ecco perché la stoffa ha qualcosa da insegnare anche alla poesia e, innanzitutto, alla loro comune attenzione verso la bellezza e la forma, quest'ultima «se prêtant [...] à des constructions, des rites, des façons d'exister qui la font s'établir et se différencier dans une société en cela meilleure» (26-27).

Inoltre, la stoffa, sottraendo il corpo alla sua sola funzionalità biologica, ha reso l'uomo consapevole della sua umanità e del suo essere anche figura, spostando così la percezione del suo esistere dall'interiorità cieca a «un dehors où l'horizon apparaît, comme lui-même une forme» (28). Grazie alla possibilità di essere divenuto testimone della sua stessa forma, l'uomo (e, con lui, la sua capacità di fare poesia) può asseverare un'unità foriera di un ordine del mondo, di un orizzonte, o, ancora meglio, aprire a «un acheminement de lumière» (Ibidem).

Infine, grazie alla caratteristica essenziale della stoffa di essere superficie in cui si stagliano simboli che richiamano alla trascendenza, che, col movimento delle pieghe, si eclissano per poi ricomparire, essa richiama in modo rassicurante al fatto che «l'intermittence est le fait de tous, et de tout» (32), non solo del divino ma anche della presenza, di cui tale discontinuità è traccia.

Si può quindi concludere, parafrasando il poeta di Tours, che senza tessuto non sia possibile cercare la verità «au plan méta-ordinaire» (35), poiché la stoffa è dialettica, come il linguaggio nella parola:

Elle ouvre à l'unité, tout autant qu'elle se referme sur l'irréalité du rêve de la personne. En fait, elle permet d'autant mieux de concevoir l'expérience de l'unité qu'elle fait aussi qu'on se perd, et quelquefois pour longtemps, dans les leurres qu'elle présente à l'esprit. Ambiguïtés dont la poésie, dans l'ordre des mots, est tout autant la pratique. (Ibidem)

Ma interessa forse a Bonnefoy cercare una verità meta-ordinaria? Di nuovo, dove e come trovare parole che sappiano dire il qui e ora della terra?

Nella seconda parte del volumetto Pensées d'étoffe ou d'argile il poeta ci parla di un ulteriore prodotto dell'uomo che ci passa fra le mani quotidianamente. Si tratta, questa volta, della tazza. Fatta d'argilla scavata nelle viscere del terre- 
no e forgiata grazie all'acqua e al fuoco, sa soddisfare una richiesta dello spirito ben più importante di quella del sogno dell'apparenza evocata dalla stoffa. La doppia funzione che gli permette, nel contempo, di preservare e celebrare, fa del «travail du potier» (38) l'equivalente del lavoro di ogni poeta che voglia sfuggire all'inganno del gioco della forma e del bel concetto:

Entre nos mains rapprochées prendre ce qui est, l'élever devant soi dans une lumière qui en devient un bien partagé, dire ce qui a valu, ce qui vaudra, célébrer. (38-39)

La stoffa ci mette comunque in guardia, al negativo, istruendoci sulle trappole del linguaggio e del pensiero concettuale, pur offrendoci, talvolta, il gusto «des chatoiements de l'imaginaire» (37) quando si è indotti ad ammirare «les plis de tissus figuratifs pour des robes qui soient par la grâce active de quelque corps le mariage de l’Un et du multiple» (37).

\section{Abiti, CORREDi, MAGLIE E MATRIMONI}

Anche in «Tout arrive», composizione poetica contenuta nella raccolta Est-ce que j'peux placer un mot? (200I) di Dominque Fourcade, si parla di robes e di mariages, nonché di lenzuola e corredi, ma con tutt'altro significato, anche se, nuovamente, il tessile è preso in considerazione per esprimersi sul metodo utilizzato in poesia.

Si tratta di un metodo che assimila il procedere del poeta a quello di tutti quegli scrittori, Proust in primo luogo, che hanno fatto della costruzione della struttura del testo una delle preoccupazioni principali del loro agire.

Com'è noto, nelle pagine conclusive della Recherche, sono rievocati tutti quegli episodi e personaggi che, come impressi sulle pieghe della stoffa citate da Bonnefoy, sono apparsi e scomparsi lungo l'intera narrazione in modi «intermittents» (À l'ombre des jeunes filles en fleur, Proust i987: t. 2, I6). Per richiamarli Proust ricorre alla metafora dei fili, della loro tessitura, del merletto e dell'abito.

Innanzitutto, la vita «entrecroise ces fils, elle les redouble pour épaissir la trame, si bien qu'entre le moindre point de notre passé et tous les autres, un riche réseau de souvenirs ne laisse que le choix des communications» (Le Temps retrouvé, Proust i987: t. 4, 607); l'opera diventa così un costante lavoro sartoriale di assemblaggio di pagine e di episodi memoriali appuntati e cuciti gli uni agli altri, come fossero «dentelle» (t. 4, 6II), di modo che, come afferma colui che ha finalmente compreso come sia divenuto scrittore, «épinglant ici un feuillet supplémentaire, je bâtirais mon livre, je n'ose pas dire ambitieusement comme une cathédrale, mais tout simplement comme une robe» (t. 4, 6ro). 
Ora, in «Tout arrive» è in corso il tentativo inedito di fare immagine in poesia senza passare né dallo schema analogico associativo, né dalla metafora, rimanendo con i piedi ben piantati a terra, benché talvolta non si veda bene dove si stia andando, come si fosse sonnambuli. Non si tessono fili, ma si procede, semmai, su funi:

nous marchons sur un câble connu de nous seuls parfois même nous marchons sur un câble qui nous est inconnu là pour le câble je savais mais rien du choc qui m'attendait - surtout nulle équivoque, pas la séduction du funambule ici - ce n'est que du somnambulisme pauvre, et le câble est enfoui - ni équivoque ni métaphore. (Fourcade 2001: 58-59)

L'incedere poetico si apre allora alla possibilità che ogni cosa della realtà sia non soltanto accolta, ma tenuta presente e richiamata in ogni momento; ci si predispone alla possibilità che qualsiasi cosa e tutto, allo stesso tempo, accada e abbia luogo. La stoffa della realtà è totalmente distesa e mise à plat sulla superficie della pagina.

Il motto «Tout arrive», che il poeta apprende essere l'intestazione della carta da lettere che Manet aveva usato per scrivere poche parole di ringraziamento a Mallarmé (57), diventa allora la chiave di volta di una poetica e di un modus operandi nuovi. In altre parole, la sovrapposizione e copresenza 'temporale', che è essenza della Recherche, si traduce nelle pagine fourcadiane in sovrapposizione e compresenza 'spaziale' di un mondo «toutarrivesque» (6I), che può essere percorso in tutti i sensi, diagonali comprese.

Così, la memoria delle immagini suggerisce essa stessa il riferimento al tessuto - ma, si badi, delle immagini concrete (dipinti, fotografie, scenografie, coreografie, pubblicità) e non delle immagini costruite per analogia emotiva. Bando all'immagine surrealista, attinta nell'amalgama dell'immaginario psichico, contro la quale tanto Ponge, quanto Bonnefoy e Fourcade, in maniera diversissima fra loro, si pronunciano.

Un tessuto che è innanzitutto linge in «Tout arrive». Pezzo di stoffa bianco, banale canotta della celebre marca «Damart» (69) che invita a «ne rien vivre sans chaleur» (Ibidem), e lenzuolo, che porta in sé, ricamate o impresse con l'inchiostro, le cifre della formula presa in prestito al pittore del Déjeuner sur l'herbe e dell' Olympia inaugurando il Moderno nella nostra epoca.

Lenzuolo che è pagina bianca, certo, ma anche tela (di lino o canapa, metonimia dello spazio del dipinto), sulle quali ogni volta qualcuno (poeta o pittore) appone una nuova firma interpretativa dell'epoca che vive, consegnandosi ogni volta in modo nuovo (pur con incertezze adolescenziali) in una durata dell'arte (poesia o pittura) che rende tutto, persino Dante, Proust o Emily Dickinson, contemporaneo: 
étonnez-vous du travail au blanc linge (ensemble des pièces de lin aux besoins du jeune ménage) ne confondez pas avec chanvre sorti de tous ces siècles pour une forme nouvelle signature de l'époque (qui dure encore) par personne (qui dure encore) quelle forme du dehors (adolescente) durée de dedans. (65)

Come ho avuto modo di osservare altrove (Riva 20I2: 69), la stoffa è, infatti, usata in alcuni passaggi di questa composizione per veicolare l'idea di trasmissione e di periodo, poiché la vera poesia e la grande arte sanno nascere ogni volta nuove, ad ogni passaggio di mano, nella loro unicità.

Un'unicità cui ogni grande artista tende e che, come una robe, un abito da sposa, non può essere preso in prestito: ognuno deve confezionare il suo perché il matrimonio sia memorabile, e occorre cercarlo, secondo Fourcade, all'interno della totalità del reale e dell'arte. Ogni risultato, ogni confezione, ogni incastellatura, ogni metodo (il «bâti de la robe de Proust»: 200r: 74) non è mai, per chi lo eredita, sufficiente e, in ogni epoca, c'è chi tenta una nuova impresa pericolosa, in cui si rischia proprio tutto, financo la vita:

Je suis sûr que seule une méthode rend plausible que tout arrive, autrement c'est infondé; l'art d'aujourd'hui va si peu de soi. Cette méthode (du bâti de la robe de Proust) je ne l'ai pas, ni Proust. Ou bien se taire en ski total, je suis persuadé qu'il y va de la planification de la mort. De toute façon tout est art. Et que je feigne quoi, je ferai ce que tu me demanderas, d'être écrivain? (74)

È per questo che con la parola linge gioca anche layette (corredino) e trousseau (corredo da sposa e, nel contempo, il baule che lo contiene), peraltro associati al lenzuolo con le cifre allacciate (maschili e femminili, che si congiungono in modo intercambiabile), in cui si evoca anche l'idea di talamo nuziale.

Tuttavia, disforicamente, esiste anche un «trousseau sans mariage» (65) nel contemporaneo, le cui cifre annodate corrispondono ad altri ruoli, quelli della vittima e del carnefice. Questo lascito, che si trasmette di generazione in generazione, porta anch'esso impresso una marcatura. Si tratta delle cifre tatuate sulle braccia dei perseguitati nei campi di sterminio (o nelle carceri di Abu Ghraib, o nelle guerre d'Algeria, del Kossovo, o sui documenti d'identità etnica nel Rwanda del I994, episodi del contemporaneo evocati in vari passi della produzione poetica fourcadiana). Gli scatti fotografici di quei corpi nudi (o torturati) sono la cifra della nostra epoca e un invito ad abbassare i toni del dibattito del secolo.

Tornando di nuovo alla questione di metodo, questi passaggi sono resi possibili dalla sovrapposizione d'immagini che, come si diceva, non traggono la loro fonte da nulla di onirico. Le associazioni si fanno su basi di realtà, benché si tratti di una realtà culturale. L'evocazione di Manet e dell'intestazione della 
sua carta da lettere evoca uno dei suoi dipinti più celebri, l'Olympia, che, a sua volta, richiama la tela di Tiziano della Venere di Urbino, in cui, in secondo piano, è proprio visibile un baule da cui una fanciulla estrae il corredo. E l'Olympia di Manet rimanda a una coreografia eponima di danza contemporanea, creata nel 1993 per Marathon for Dance da Vera Mantero, un assolo di I7 minuti durante il quale la danzatrice legge passi tratti da L'asphixiante culture di Jean Dubuffet. L'incarnazione ossuta della fanciulla adagiata nuda su un lenzuolo, che guarda dritta negli occhi lo spettatore mentre riflette sul ruolo politico della cultura, è epifania delle immagini dei corpi nudi dei lager.

Ulteriore elemento della poetica fourcadiana relativo alla costellazione semantica del vêtement, l'ultimo che si prenderà in considerazione, è non meno importante della «robe» (e della maglia Damart): si tratta di «doublure» $(67)$, che, fra le tante cose che può significare (quali doppiaggio o rimpiazzo in uno spettacolo teatrale) significa soprattutto fodera: ciò che è consustanziale al vestito, ma che non può, né deve essere visto quando lo si indossa. La «doublure» è, secondo Fourcade (Riva 20I2: 93-94), la realtà stessa e il modo di porgerla. In un certo senso, si può dire che per Fourcade si tratti del suo stesso metodo poetico. Un metodo che gioca retoricamente con stratificazioni di endiadi e isocolon (Fourcade 200I: 67), ossia con sdoppiamenti e forme di duplicazione e parallelismi fra sensi e suoni, fra immagini e luoghi diversi tutti strettamente attinenti alla sfera del reale, fra spazi pieni e spazi vuoti, fra dentro e fuori che, come nei dipinti di stoffa di Simon Hantaï (cui Fourcade dedica molti riferimenti lungo gran parte della sua produzione poetica, nonché la curatela di una grande retrospettiva al Centre Pompidou nel 2013) ${ }^{4}$, sanno dire «l'envers des choses, ou l'inséparable contraire» (Didi-Huberman I998: I23), mentre «enkyste[nt] un temps [et] accouche[nt] d'un espace» (I24).

\section{IN GUISA DI CONCLUSIONE}

Une duite est un fil que la navette d'un métier à tisser conduit depuis une lisière jusqu'à l'autre, dans l'ourdissage d'une étoffe quelconque. Létymologie de ce mot réside dans l'ancien verbe duire, qui signifie conduire. (Didi-Huberman I998: I9, corsivi dell'autore)

Passando per tele di ragno, orditi, jeans, divise maoiste, canottiere, corredi e abiti nuziali, è possibile concludere che la stoffa, in ognuno dei poeti qui brevemente analizzati, è stata usata come mezzo per esporre non solo la pratica o la riflessione sul metodo di condotta del fare poetico (in riferimento, rispettivamente, ai temi della necessità del lavoro di ritessitura da

4 Vedasi il catalogo Simon Hantaï (Fourcade-Monod-Fontaine-Pacquement 20I3). 
parte dei lettori, del rischio insito nell'uso del linguaggio se esso si limita a cogliere la bellezza dell'apparenza, della capacità delle rappresentazioni visive concrete di fare immagine in poesia), ma anche sull'intrinseca doppiezza della sua materia.

In particolare e, di nuovo rispettivamente, Ponge asserisce, al ritmo di una sarabanda, l'indissolubilità, se non il prevalere della fisicità della parola sul significato, Bonnefoy esprime l'ambivalenza di quel linguaggio che dice la forma senza affermare la presenza, Fourcade concretizza il galleggiamento e la stratificazione della parola-immagine moltiplicando effetti e giochi di suono-senso diagonali per trovare e realizzare a sua volta, così come Proust, un bâti.

In un passo giovanile, questa volta poetico, Yves Bonnefoy afferma che solo le stoffe macchiate possono trionfare su quelle perfette dei poeti trafficanti di eterno5.

In queste, e non nelle altre, la realtà mostra il suo metodo obliquo e la sua assenza di 'uno' sguardo, che è il solo modo di vedere e di procedere della poesia, nel contemporaneo.

\section{RIFERIMENTI BIBLIOGRAFICI}

Bonnefoy Y., I978, «Anti-Platon», in Poèmes, Paris, Mercure de France, (I947).

—, I989, Sur un sculpteur et des peintres, Paris, Plon.

—, 20Iо, Pensées d'étoffe ou d'argile, Paris, Éditions de L'Herne (Carnets).

Dictionnaire de la langue française «Littré», en ligne: http://littre.reverso.net/dictionnaire-francais/.

Didi-Huberman G., I998, L'Étoilement, sur Simon Hantaï, Paris, Minuit.

Dubuffet J., I968, Asphixiante Culture, Paris, J.J. Pauvert.

Fourcade D., 200I, «Tout arrive», in Est ce que j'peux placer un mot?, Paris, P.O.L.

Fourcade D.-Monod-Fontaine I.-Pacquement A. (eds.), 20I3, Simon Hantaï, Paris, Éditions du Centre Pompidou.

Hugues P., I996, Tissu et travail de civilisation, Rouen, Médianes.

Maulpoix J.-M., 2002, Le Poète perplexe, Paris, Corti.

Meadows P.A., I997, Francis Ponge and the Nature of Things: From Ancient Atomism to a Modern Poetics, Cranbury, Associated University Press.

Ponge F., I96I, «Le monde muet est notre seule patrie», in Le Grand Recueil, t. 2: Méthodes, Paris, Gallimard.

—, I999, «L'Araignée», in Pièces, Euvres complètes, B. Beugnot (ed.), Paris, Gallimard (Bibliothèque de la Pléiade).

5 «Toutes choses d'ici, pays de l'osier, de la robe, de la pierre, c'est-à-dire: pays de l'eau sur les osiers et les pierres, pays des robes tachées. Ce rire couvert de sang, je vous le dis, trafiquants d'éternel, visages symétriques, absence du regard, pèse plus lourd dans la tête de l'homme que les parfaites Idées, qui ne savent que déteindre sur sa bouche» (Bonnefoy I978: II). 
Proust M., I987, À la recherche du temps perdu, J.-Y. Tadié (ed.), Gallimard, Paris (Bibliothèque de la Pléiade).

Riva S., 2012, Gli scandali di Empedocle. Note a margine della traduzione di «Tout arrive» di Dominique Fourcade, in D. Fourcade, Tutto accade / Tout arrive, Milano, La Vita Felice: 49-IoI.

Scheid J.-Svenbro J., I994, Le métier de Zeus: mythe du tissage et du tissu dans le monde gréco-romain, Paris, La Découverte.

Svenbro J., 2008, Figures textiles de la parole dans l'Antiquité gréco-romaine, in L. André-O. Blanc-F. Charpigny (eds.), Textes et textiles du Moyen Âge à nos jours, Lyon, ENS éditions (Métamorphoses du livre). 